\title{
Estimation of Electrical Pathways Finding Minimal Cost Paths from Electro-Anatomical Mapping of the Left Ventricle
}

\author{
Rubén Cárdenes ${ }^{1,2 \star}$, Rafael Sebastian ${ }^{3}$, David Soto-Iglesias ${ }^{1}$, David Andreu ${ }^{4}$, \\ Juan Fernández-Armenta ${ }^{4}$, Bart Bijnens ${ }^{1,5}$, Antonio Berruezo ${ }^{4}$, and Oscar \\ Camara $^{1}$ \\ 1 PhySense, DTIC, Universitat Pompeu Fabra, Barcelona, Spain, \\ 2 Fetal and Perinatal Medicine Research Group, IDIBAPS, Hospital Clinic de \\ Barcelona, Spain \\ 3 Computational Multi-Scale Physiology Lab, Universitat de València, Spain \\ 4 Arrhythmia Section, Cardiology Department, Thorax Institute, Hospital Clinic de \\ Barcelona, Spain \\ 5 ICREA, Barcelona, Catalonia, Spain
}

\begin{abstract}
The electrical activation of the heart is a complex physiological process that is crucial for the understanding of several cardiac dysfunctions, such as ventricular tachycardia (VT). Nowadays, electro anatomical mappings of patient-specific activation times on the left ventricle surface can be estimated, providing important information to the clinicians for cardiac treatment. However, true relations with Purkinje activations, or relevant conduction channels are difficult to detect. We present here a novel method to find electrical pathways using minimal cost paths computations on surface maps. The results of this method have been carried out in simulated data, showing the validity of our method, and also in clinical data, showing important information about the pathways and relating them with main conduction channels present in the left ventricle.
\end{abstract}

Key words: electrical pathways, Purkinje, streamlines, fast marching, singular points, electro-anatomical mapping, cardiac arrhythmias, ventricular tachycardia

\section{Introduction}

Ventricular tachycardia (VT) is one type of severe cardiac arrhythmias which is often treated with Radio-Frequency Ablation (RFA) techniques. The planning of these interventions has been substantially improved during last years with the integration of patient-specific imaging data with invasive electro-anatomical mapping $([1,2])$ helping on better targeting ablation sites. Nevertheless, some VTs

* ruben. cardenes@upf.edu 
are induced by patho-physiological mechanisms for which very limited patientspecific data is available such as abnormalities of the cardiac conduction system (CCS) in fascicular VTs.

The CCS is a heterogeneous network of cells responsible for the fast and coordinated distribution of the electrical impulses that triggers the contraction of the heart. In the ventricles, the CCS is composed of the His bundle (HB) and bundle branches (BB) that are connected to the most distal section, often called Purkinje (PK) system (see Fig. 1 left). The PK system plays a key role in the synchronous activation of the ventricles since it dictates the starting points of activation and therefore the electrical activation sequence [3]. Unfortunately, the CCS cannot be fully extracted from in vivo images because the size of its structures is in the order or cellular resolution. Nevertheless, researchers have recently developed generic computational models capturing PK tree-like structure from ex vivo data available for different species. The reader is referred to [2] for a review of these computational models.

In severe fascicular VTs, the RFA intervention is based on the ablation of PK end-terminals (junctions between the CCS and the myocardial muscle) which are identified by manual detection of PK activations (fast deflections of small amplitude prior to the main electrocardiogram complex in 1D signals acquired with patient-specific electro-anatomical mapping). Unfortunately, the detected PK end-terminals do not provide information about the whole tree-like structure of the CCS. We present here a methodology to obtain patient-specific activation lines from electro-anatomical maps based on finding geodesic paths. The methodology is a three-step procedure: first, the end terminals are detected from the electro-anatomical map; second, the electrical paths going from the detected end-terminals are reconstructed from the electro-anatomical map; and third, the conduction velocity of the geodesic paths are computed, to distinguish those that are close to the PK system from those that are only due to muscle activation.

For validation purposes we performed two experiments on synthetically generated electro-anatomical mappings. The first one is a simplistic simulation based on a surface fast marching solution to show the behavior of the geodesics generation. The performance of the detection of the end-terminals is evaluated with a second experiment where a simulated local activation map (LAT) is obtained for every point using detailed electrophysiological models and including a PK tree constructed with a L-systems-like method [2] on a LV geometry extracted from a CT image. Finally, we applied the algorithm to a real case of a patient with fascicular TV, finding relevant information about PK terminal distribution for the planning of ablation procedures.

\section{Methods}

\subsection{Minimal cost paths}

Minimal cost path computation is a well-known problem that is usually solved

by Dijkstra algorithm [4] in graphs. For 2D/3D images, the pixels or voxels are 
used as the nodes and the distances between them are the weights associated to the edges of the graph. Computation of geodesics on surfaces has a higher level of complexity that has been addressed by other authors $[5,6]$ in the past. In particular, given a surface $\mathcal{S}$, with a weighted map $\psi$ defined on it, $\psi(\boldsymbol{x}) \forall \boldsymbol{x} \in \mathcal{S}$, and a surface point $\boldsymbol{z}$ denoted as the end point, the geodesic problem consists in finding the shortest weighted path $\Gamma_{\psi} \boldsymbol{y}:[0,1] \rightarrow \mathcal{S}$ lying on the surface between any point on the surface $\boldsymbol{y}$ and $\boldsymbol{z}$ :

$$
\Gamma_{\psi, \boldsymbol{y}}=\underset{\gamma}{\operatorname{argmin}}\left\{L_{\psi}(\gamma): \gamma(0)=\boldsymbol{y}, \gamma(1)=\boldsymbol{z}\right\}
$$

where $L_{\psi}(\gamma)$ is the curve weighted length

$$
L_{\psi}(\gamma)=\int_{0}^{1} \psi(\gamma(s))\left\|\gamma(s)^{\prime}\right\| d s
$$

It is known $[5,7]$ that a solution to this problem can be obtained using a hamilton-jacobi formulation, solving the eikonal equation for $\phi(\boldsymbol{x})$, that will define a distance map on the surface starting from $\boldsymbol{z}$

$$
\|\nabla \phi(\boldsymbol{x})\|=1 / \psi(\boldsymbol{x}), \forall \boldsymbol{x} \in \mathcal{S},
$$

with initial condition $\phi(\boldsymbol{z})=0$. The solution to this equation is optimally computed using the fast marching algorithm [8]. Once $\phi$ is computed, the computation of the geodesic can be reformulated as a backtracking procedure following the gradient of $\phi$

$$
\Gamma_{\psi, \boldsymbol{y}}=\left\{\boldsymbol{x} \in \mathcal{S} \mid \nabla \Gamma_{\psi, \boldsymbol{y}}(\boldsymbol{x})=\nabla_{\mathcal{S}} \phi(\boldsymbol{x}), \Gamma_{\psi, \boldsymbol{y}}(0)=\boldsymbol{y}, \Gamma_{\psi, \boldsymbol{y}}(1)=\boldsymbol{z}\right\},
$$

where $\nabla_{\mathcal{S}} \phi(\boldsymbol{x})$ denotes the gradient of $\phi$ intrinsic to the surface. This means that starting from any point $\boldsymbol{y}$, the geodesic on the surface induced by $\psi$ is obtained following the gradient of $\phi$. Two considerations have to be taken into account. First, the surface $\mathcal{S}$ has to be a good approximation of a Riemmanian manifold, in other words, be smooth enough to allow computing its gradient. Secondly, $\nabla_{\mathcal{S}} \phi(\boldsymbol{x})$ has to be defined for all $\boldsymbol{x}$ on $\mathcal{S}$. Singular points, such as sink or source points are excluded from the path computation, however, they will provide important information as we will show in the next section. Notice that if the weighted map $\psi$ is constant, we are in the Euclidean case. However, it is interesting for other applications the use of any other maps defined on the surface. In our case, $\phi$ is directly given by the LAT map, where $\boldsymbol{z}$ is located at the HIS, and therefore, direct application of equation 1 (i.e. backtracking) will provide the geodesics. Notice that the geodesics will be calculated from late local activation times to early activation times, thus using the negative of the gradient field, $-\nabla_{\mathcal{S}} \phi$. Our implementation will follow the description given in [7], where the computations are performed on implicit surfaces instead of on triangular meshes. 


\subsection{End-terminals detection}

The end-terminals are estimated directly from the electro-anatomical maps. Observing closely the gradient field of the electro-anatomical maps (Fig. 1 right) one can clearly distinguish points where the geodesics converge or diverge, that are singular points of the map, or points where the gradient field of the map is not well defined. Therefore, a point $\boldsymbol{x}$ on a distance map $\phi$ defined on the surface $\mathcal{S}$ will be detected as a singular point if the lateral derivatives of the gradient map are different at every tangential direction. In practice, the singular points are detected when the sum of these derivates are below a certain threshold:

$$
\lim _{h \rightarrow 0^{+}} \frac{\nabla_{u_{i}} \phi(\boldsymbol{x})-\nabla_{u_{i}} \phi\left(\boldsymbol{x}-u_{i} h\right)}{h}+\lim _{h \rightarrow 0^{-}} \frac{\nabla_{u_{i}} \phi(\boldsymbol{x})-\nabla_{u_{i}} \phi\left(\boldsymbol{x}-u_{i} h\right)}{h}<\varepsilon,
$$

where $u_{i}$ are the coordinates of the tangential plane of $\mathcal{S}$ defined on $\boldsymbol{x}$, and $\nabla_{u_{i}} \phi$ the gradient component in this direction. The threshold selection will be studied in section 3.2.

A distinction has to be made between two types of singular points, those where geodesics converge, denoted as sink points (point A in Fig. 1) and source points where geodesics diverge (point B in Fig. 1). These points can be discriminated based on the change of sign of $\nabla_{u_{i}} \phi$ along each tangential direction. A change from positive to negative give sink points and source points otherwise. Our points of interest here are the sink points of the activation map $\phi(\boldsymbol{x})$, that will be equivalent to source points of the negative map $-\phi(\boldsymbol{x})$.
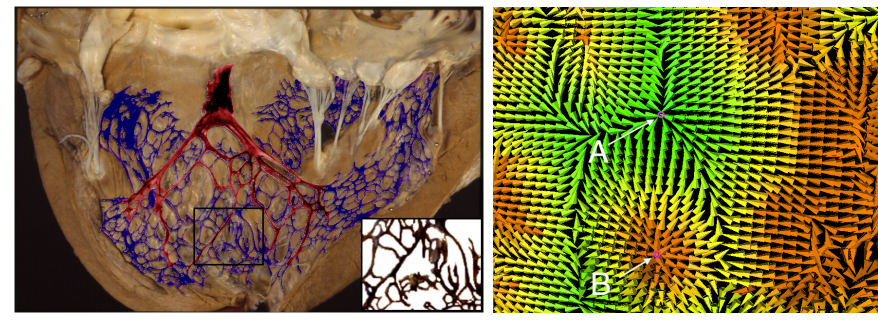

Fig. 1. Left: detail of the Purkinje fiber system in a real case. Right: vector field of an electrical activation map. Two singular points are indicated with arrows, a sink point: A, and a source point: B.

\section{Validation experiments with simulated data}

\subsection{Fast marching generated simplistic map}

To show the behavior of the proposed technique we have designed a simple simulation experiment with synthetic data. Notice that the simulated map is not meant to be realistic but it is designed to show the tracking method performance. 
We have taken a surface model of a left ventricle, $\mathcal{S}$, and a tree mimicking its main PK system defined on it. Starting from a tree point located at the HIS, a signal is propagated to the rest of points in the PK tree with a constant velocity $v_{1}$. Starting from the local activation times obtained on this tree, a signal is propagated using fast marching to the rest of the surface points with a lower velocity, $v_{2}=v_{1} / 10$, obtaining a simulated activation map $\phi(\boldsymbol{x}) \forall \boldsymbol{x} \in \mathcal{S}$. Fig. 2 (left) shows the reference tree with the surface mapped with the local activation times obtained in this way. Our goal in this experiment is to use this map to recover the initial tree with the backtracking algorithm described above. In Fig. 2 (middle), we show the geodesic paths obtained starting from a set of seeds equally distributed on the surface (2478 seeds in total). This give us information about many possible activation paths on the ventricle. Then, calculating the velocity along the generated paths and keeping the ones with high velocities we are able to recover the original PK tree as shown in Fig. 2 (right). We have then computed the LAT map from the computed tree to compare it with the original LAT map. This map is shown in figure Fig. 2 (middle) and the absolute differences between both are shown in Fig. 2 (right), wiht a maximum difference of $25 \mathrm{~ms}$ and an average relative error of $5.20 \%$. This figure shows that the bigger differences are obtained in the basal part, with low errors for the rest.
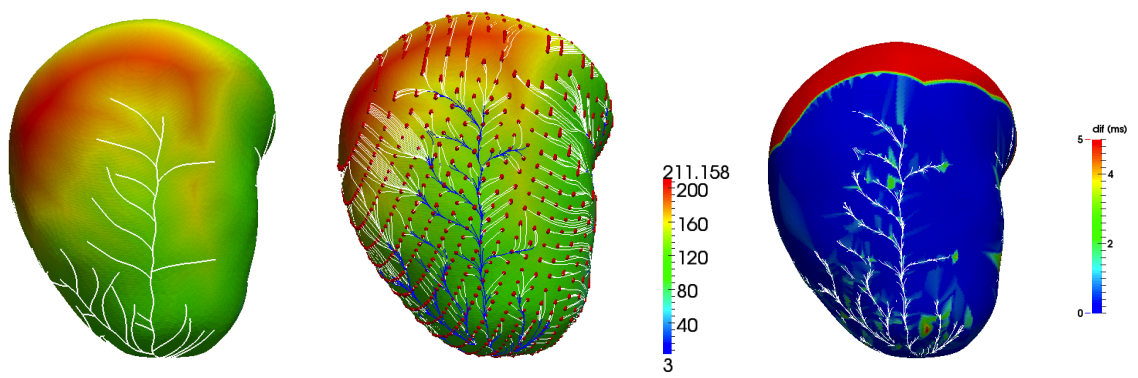

Fig. 2. Ventricle surface with simulated activation maps. Left: a reference (simulated) PK tree is shown from which the activation map is computed. Middle: geodesic paths computed from the simulated map using uniformly distributed seeds over the surface (shown in red), and map generated from the filtered tree. Right: differences between initial LAT and LAT computed with the obtained filtered tree, shown in white.

\subsection{Realistic simulated data}

A realistic simulation of the electrical maps has been generated using the method described in [2]. The method is an enhancement of a rule-based method known as the Lindenmayer systems (L-systems). The construction of the PK tree is divided into three consecutive stages, which subsequently develop the CCS from proximal to distal sections. Each stage is governed by a set of independent user 
parameters together with anatomical and physiological constrains to direct the generation process and adhere to the structural observations derived from histology studies. Several properties of the tree are defined using statistical distributions to introduce stochastic variability in the models. The CCS built with this approach can generate electrical activation sequences with physiological characteristics. The electrical propagation in the myocardium was modeled using the monodomain equation.

Using these maps, we have tested our end-terminals detection, by finding the singular points, and comparing with the reference points from the simulated experiment. Fig. 3 (left) shows the simulated activation map in a left ventricle model where the end-terminals are shown. The end-terminals detected with our method are shown in Fig. 3 (middle). For quantitative evaluation, the distances from the detected points and the reference points are computed. To account for under or overestimation of the detected points with respect to the threshold used, two distance measures are computed, the average distance computed from each reference point to its nearest detected point, and viceversa, the average distance computed from each detected point to its nearest reference point. The optimal threshold value is taken when these two distances reach low values at the same time. From Fig. 3 (right) the optimal threshold is found at approximately 0.35 , providing average distances of 0.98 and $0.88 \mathrm{~mm}$ respectively.
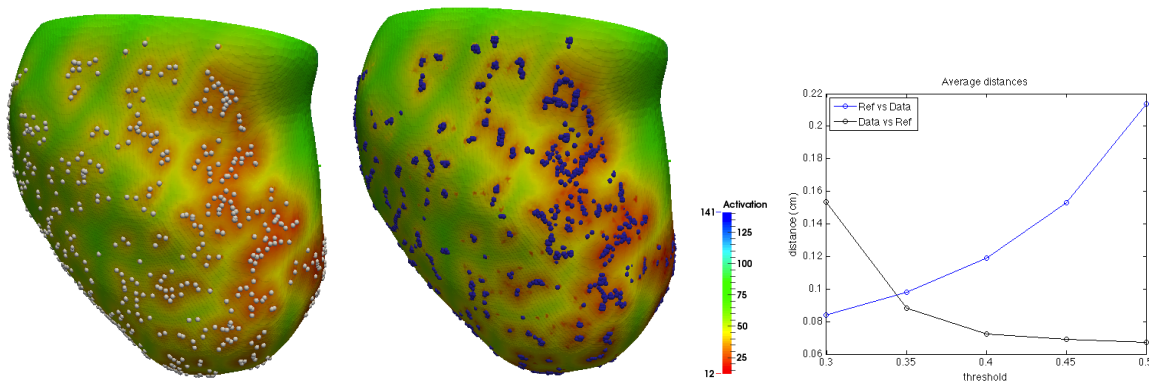

Fig. 3. Left: synthetically generated electrical map using [2], with end-terminals shown in white. Middle: automatically detected end-terminals shown in blue for $\varepsilon=0.35$. Left: Average differences between the reference end-terminals and the detected singular points and viceversa vs the threshold value, $\varepsilon$.

\section{Clinical data results}

For real cases, we had access to CT images to extract the LV geometry and electro-anatomical mapping data giving electrical information at the endocardium of the LV, for one case having fascicular VT. The mapping of the CARTO data onto the CT geometry was based on establishing an homeomorphism between both surfaces using a common parameterization computed by mesh flattening 
(see details in [9]). The electro-anatomical maps have 231 points with a 1D electrocardiogram signal (acquired at $1 \mathrm{kHz}$ ) where Purkinje and muscle activations were visually identified by an experienced technician in Hospital Clínic de Barcelona. The surface mesh used in this case has 50.000 points, with LAT values interpolated linearly.

Fig. 4 shows the geodesic paths automatically computed for this case, generated from the singular sink points detected by our method from the negative gradient field of the activation map, $-\nabla_{\mathcal{S}} \phi$. The $\mathrm{LV}$ is colored with the activation map in two different views. The geodesic paths have also been colored according to the velocity, see Fig. 4 (E, F), in the same views for comparison. Notice that a discrimination between fast or slow conduction channels is difficult or impossible in the clinical case because the conduction velocity considerably changes along every path. However, it is interesting to see regions with consistently higher velocity, giving a hint about possible contacts with the PK system.
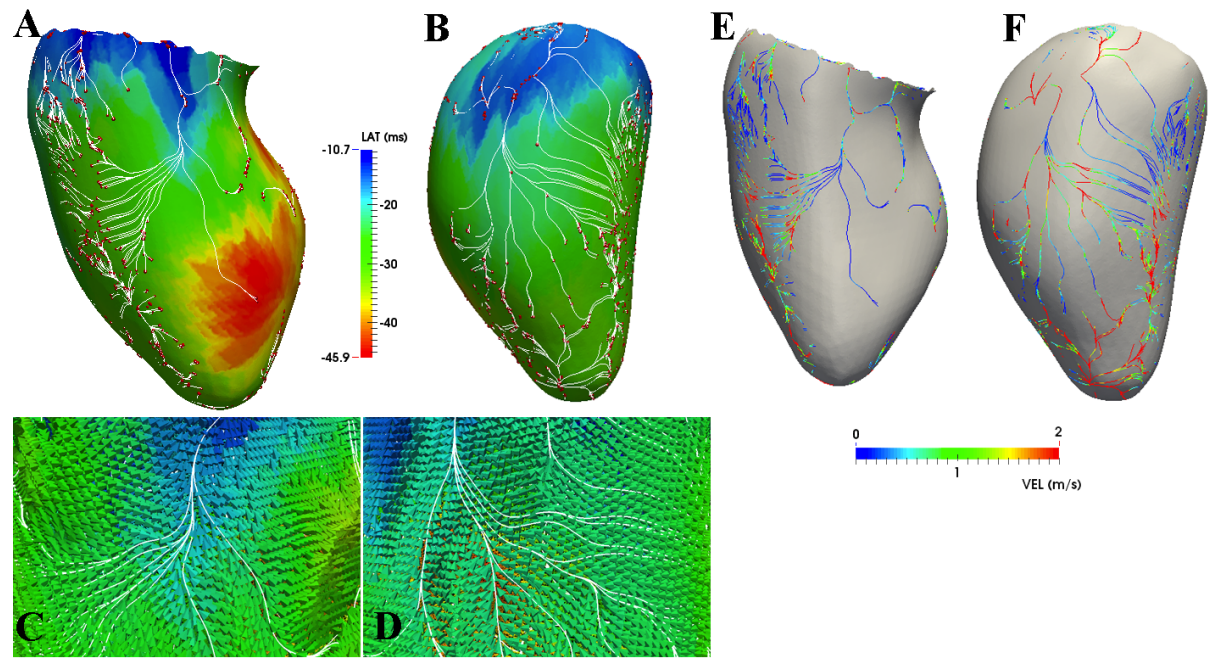

Fig. 4. A, B: LV surface from a real patient with overlaid measured activation maps, computed geodesic paths (white), and seeds (red). C, D: detail of the geodesic paths with the gradient vector field obtained from the activation maps. E, F: geodesics colored according to their conduction velocity

\section{Conclusions}

We have proposed a method to automatically trace the electrical pathways in activation maps of the LV surface. It is important to remind that these electrical pathways are just the pathways of electrical activation through the muscle, and are not meant to be an exact reconstruction of the PK system. Taking this 
into account, the geodesic paths obtained here have several advantages over the electrical maps. First, they provide an attractive and an alternative visualization to the activation maps, giving more detailed local information. For instance, we can better see channels forming loops, or branching systems, that are sometimes hidden by the global color-coded maps, and that are useful to the clinicians to understand and treat abnormal electrical patterns. Second, the velocity information along the geodesic paths give us an approximate idea about how is the distribution of the PK system, providing information about the connections of the conduction paths of the muscle to the PK system. The geodesics shown here open the possibility for better visualizations in other cases of VT and can be used as an alternative way to identify scar tissues.

\section{Acknowledgements}

This work is partially funded by the Sub-programa de Proyectos de Investigación en Salud Instituto de Salud Carlos III, Spain (FIS - PI11/01709), by Spanish Ministry of Science and Innovation (TIN2011-28067), and by eTorso project (2013-001404) from Generalitat de Valencia.

\section{References}

1. Piers, S., van Huls van Taxis, C., Tao, Q., van der Geest, R., Askar, S., Siebelink, H.M., Schalij, M., Zeppenfeld, K.: Epicardial substrate mapping for VT ablation in patients with nonischaemic cardiomyopathy: a new algorithm to differentiate between scar and viable myocardium developed by simultaneous integration of CT and contrast-enhanced MR imaging. European Heart J. 34 (2013) 586-596

2. Sebastian, R., Zimmerman, V., Romero, D., Sanchez-Quintana, D., Frangi, A.: Characterization and modeling of the peripheral cardiac conduction system. IEEE Trans Med Imaging 32(1) (Jan 2013) 45-55

3. Durrer, D., van Dam, R.T., Freud, G.E., Janse, M.J., Meijler, F.L., Arzbaecher, R.C.: Total excitation of the isolated human heart. Circulation 41(6) (Jun 1970) 899-912

4. Dijkstra, E.: A note on two problems in connection with graphs. Numerische Math. 1 (1959) 269-271

5. Kimmel, R., Sethian, J.A.: Computing geodesic paths on manifolds. Proc. Nat. Acad. Sci. 95(15) (1998) 8431-8435

6. Mart'ınez, D., Velho, L., Carvalho, P.: Computing geodesics on triangular meshes. Computers \& Graphics 29(5) (Oct 2005) 667-675

7. M'emoli, F., Sapiro, G.: Fast computation of weighted distance functions and geodesics on implicit hyper-surfaces. Journal of Computer Physics 173 (2001) 764 730

8. Sethian, J.A.: A fast marching level-set method for monotonically advancing fronts. Proc. Nat. Acad. Sci. 93 (1996) 1591-1595

9. Soto-Iglesias, D., Butakoff, C., Andreu, D., Fernández-Armenta, J., Berruezo, A., Camara, O.: Evaluation of different mapping techniques for the integration of electro-anatomical voltage and imaging data of the left ventricle. In: Functional Imaging and Modeling of the Heart. Volume 7945., London, UK, LNCS (2013) 391399 